\title{
INFLUENCIA DE UN PROGRAMA DE PSICOPROFILAXIS DEL CLIMATERIO EN LA SINTOMATOLOGÍA CLIMATÉRICA DE MUJERES ATENDIDAS EN UN HOSPITAL DE HUARAZ, 2016
}

\author{
INFLUENCE OF A CLIMATERIO PSYCHOPROPHILAXIS PROGRAM IN THE CLIMATRIC SYMPTOMS OF \\ WOMEN ATTENDED AT A HUARAZ HOSPITAL, 2016
}

Flora Flores-Tiznado (iD) $1, a$

\begin{abstract}
Objetivo: Evaluar la influencia de la aplicación de un programa de psicoprofilaxis del climaterio en la intensidad de la sintomatología de mujeres climatéricas atendidas en el Hospital "Nuestra Señora de las Mercedes" de Carhuaz, en Huaraz, durante el año 2016. Material y métodos: Se realizó un estudio cuasi experimental, en una muestra total de 30 mujeres donde el grupo experimental y el control presentaron igual proporción. Se implementaron 5 sesiones de psicoprofilaxis del climaterio dictado por los obstetras. Se utilizó el cuestionario de la escala cubana para clasificar la intensidad de la sintomatología del climaterio. La información se procesó mediante el programa SPSS, utilizándose para el análisis la prueba de diferencia de proporciones y el chi cuadrado. Resultados: Luego de la intervención se evidenció un aumento en el número de mujeres asintomáticas con respecto a los síntomas vasomotores $(33,4 \%)$, psicológicos $(40 \%)$, genitourinarios $(36,7 \%)$, generales $(40 \%)$ y clínicos $(36,7 \%)$; encontrando que el programa propuesto influyó significativamente $(p<0.05)$ en la reducción de la sintomatología en todas las dimensiones estudiadas. Conclusión: Se concluye que la aplicación del programa de psicoprofilaxis del climaterio influye significativamente en la reducción de la intensidad de la sintomatología de mujeres climatéricas en el Hospital "Nuestra Señora de las Mercedes" de Carhuaz.
\end{abstract}

Palabras clave: Programa, Psicoprofilaxis del climaterio, Sintomatología (Fuente: DeCS, BIREME)

Objective: To evaluate the influence of the application of a climacteric psychoprophylaxis program on the intensity of symptomatology of climacteric women treated at the "Nuestra Señora de las Mercedes" Hospital in Carhuaz, in Huaraz, during 2016. Material and methods: A quasi-experimental, prospective, and comparative study was carried out in a sample of 30 women for each group (experimental and control). The questionnaire of the Cuban scale was used to classify the intensity of the climacteric symptomatology. The information was processed through the SPSS program, using the proportions difference test and the chi square for the analysis. Results: After the intervention, there was an increase in the number of asymptomatic women with respect to vasomotor (33.4\%), psychological (40\%), genitourinary (36.7\%), general (40\%) and clinical symptoms. (36.7\%); finding that the proposed program significantly influenced $(p<0.05)$ in the reduction of symptomatology in all the dimensions studied. Conclusion: It was concluded that the application of the climacteric psychoprophylaxis program significantly influences the reduction of the intensity of climacteric women's symptoms in the "Nuestra Señora de las Mercedes" Hospital in Carhuaz.

Key words: Program, Climateric psychoprophylaxis, Symptomatology (Source: MeSH NLM)

Filiación:

1. Universidad Nacional Santiago Antúnez de Mayolo.

Obstetra. Doctora en Ciencias de la Salud.
Citar como: Florez-Tiznado F. Influencia de un programa de psicoprofilaxis del climaterio en la sintomatología climatérica de mujeres a tendidas en un hospital de Huaraz, 2016 Revista Internacional de Salud Materno Fetal. 2020; 5 (3): 10-18. DOI: https://doi.org/10.47784/rismf.2020.5.3.89
her
Financiamiento: Autofinanciado por los autores

Conflictos de interés: La autora declara no presentar conflictos de interés.

Correspondencia: Flora Flores-Tiznado (florant298@hotmail.com)

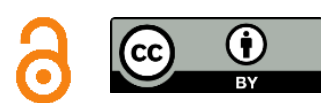

Recibido: 23 de Agosto del 2020 Aprobado: 25 de Septiembre del 2020 Publicado: 30 de Septiembre del 2020 


\section{INTRODUCCIÓN}

El profesional en Obstetricia, como parte de su formación académica, aprende y desarrolla la construcción del conocimiento acerca de los cambios de la mujer en cada etapa de su vida, con énfasis en los cambios que ocurren al cesar su función reproductiva (1).

El climaterio es el periodo de transición desde la etapa reproductiva hasta la no reproductiva y abarca, aproximadamente, desde un año antes (cuando comienzan a presentarse cambios hormonales $y$ físicos) hasta un año después de la menopausia (2), motivo por el cual aunque se constituye en un evento fisiológico en la vida de la mujer, se presenta acompañado de diversas modificaciones de tipo orgánico y psicológico, algunas de inicio inmediato y otras de instauración tardía, que en su conjunto constituyen el síndrome climatérico (3-5). Los reportes internacionales indican que la edad media de presentación de la menopausia es de 48,5, valor similar al de Perú donde gran parte de ellas refieren algún tipo de sintomatología (síntomas urogenitales, vasomotores y psicológicos) con un efecto importante en su experiencia sexual, situación que se atribuye al estado de hipoestrogenismo $(6,7)$.

Los sofocos se constituyen como el síntoma más frecuente de la menopausia, los mismos que son percibidos por el $86 \%$ de las mujeres, provocando enrojecimiento súbito de la piel, seguido de un calor del cuerpo intenso y transpiración; así mismo también se presentan síntomas psicológicos tales como la depresión, la ansiedad, la tristeza o irritabilidad (8). Las mujeres latinoamericanas presentan una sintomatología más intensa que las de otros continentes (9), tal y como lo evidenció Schnatz en su estudio llevado a cabo en Estados Unidos, donde comparó la presencia de síntomas climatéricos entre mujeres caucásicas e hispánicas, y encontró que estas últimas presentan síntomas severos (10-12).

En Cuba hace más de 20 años se creó el grupo de climaterio y menopausia y una de sus primeras tareas fue concebir un manejo interdisciplinario (13). Asimismo, diversas investigaciones en Europa y el Brasil indican que las mujeres activas físicamente presentan las frecuencias más bajas en la intensidad de los síntomas psicológicos y vasomotores $(14,15)$ en comparación con las mujeres sin actividad física. En general, la evidencia sobre la influencia de programas educativos basados en la actividad física sobre los síntomas climatéricos proviene de estudios de intervención, los cuales reportan una reducción en la frecuencia de síntomas vasomotores y la sintomatología asociada (16-19), así como una mejora en la salud psicológica y la calidad de vida (20). En otro estudio, el yoga también se reveló como una actividad beneficiosa (21).

Escasas investigaciones a nivel nacional han destacado la importancia de la implementación de un programa de psicoprofilaxis del climaterio; el cual está destinado tanto a la prevención como a la intervención en la salud pública. En aquellas instituciones donde se lleva a cabo sin la implementación adecuada. El Hospital "Nuestra Señora de las Mercedes de Carhuaz", en el cual se atiende una cantidad significativa de mujeres mayores de 45 años (22), requiere la realización de investigaciones que evalúen la aplicación de un programa, a fin de brindar una buena educación que permita mejorar notablemente la sintomatología de las mujeres climatéricas. Por ello se propone como objetivo evaluar la influencia de la aplicación de un programa de psicoprofilaxis del climaterio en la intensidad de la sintomatología de mujeres climatéricas atendidas en el Hospital "Nuestra Señora de las Mercedes" de Carhuaz durante el año 2016.

\section{MATERIAL Y MÉTODOS}

De acuerdo con la naturaleza del problema, la investigación fue cuasiexperimental. La población estuvo constituida por 216 mujeres climatéricas del Hospital "Nuestra Señora de las Mercedes" de Carhuaz durante el año 2016, establecimiento de salud de categoría 2-1, ubicado en el distrito de Carhuaz, provincia de Carhuaz, región Ancash; en donde se seleccionaron para el grupo experimental a las mujeres climatéricas atendidas en el Servicio de Obstetricia y para el grupo control a aquellas mujeres climatéricas atendidas el Servicio de Ginecología. Se excluyeron a las mujeres que presenten algún impedimento físico o mental para su participación.

Las actividades educativas propuestas estuvieron conformadas por 5 sesiones que tuvieron un tiempo de duración de 90 minutos cada sesión y fueron 
brindadas por los profesionales del servicio de obstetricia, quienes brindaron información acerca de la psicología, sexualidad y salud durante el climaterio. Las presentes actividades no se encuentran normadas por el Ministerio de Salud, aunque se han venido proponiendo en centros de salud de la región, sin embargo, ninguno ha evaluado estadísticamente la influencia que estas actividades presentan sobre la sintomatología de la mujer climatérica.

El cálculo del tamaño de la muestra consideró una proporción esperada de $44 \%$ mujeres climatéricas con ausencia de sintomatología a pesar del climaterio, la cual fue estimada en base a las observaciones de la práctica clínica, y una proporción esperada de $90 \%$ de mujeres climatéricas con sintomatología severa a causa del climaterio. Considerando un nivel de confianza del $95 \%$ y un poder estadístico del $80 \%$ se obtuvo un total de 15 mujeres climatéricas en cada uno de los grupos (control y experimental) haciendo un total de 30 participantes.

Se utilizó la Escala Cubana para Clasificar la Intensidad de la Sintomatología del Climaterio, la cual es un instrumento validado internacionalmente (23) que constó de 28 ítems a evaluar, divididos a su vez en 5 subescalas: i) Síntomas vasomotores, ii) Síntomas psicológicos, iii) Síntomas genitourinarios, iv) Síntomas generales y v) Signos clínicos.

El instrumento de recolección de datos fue sometido a la prueba de Juicio de Expertos para su validación, para lo cual se consultó a tres profesionales en Obstetricia en la especialidad de Salud Sexual y Reproductiva, un Médico GínecoObstetra y una Psicóloga, quienes mostraron todos estar de acuerdo con el contenido del instrumento. Luego de la aplicación de una prueba piloto al $10 \%$ de la muestra total, se procedió al cálculo de la confiabilidad de los instrumentos mediante el Coeficiente alfa de Cronbach, el cual presentó un valor final de 0.816 .

Para el procesamiento de la información y de acuerdo con la naturaleza de las preguntas, se usó el programa estadístico SPSS V21.0. Se utilizó como instrumento de análisis la distribución de frecuencias por porcentajes, cuadros de doble entrada y gráficos de frecuencia. Por otra parte, las pruebas de análisis estadístico que nos permitieron establecer la influencia de la variable independiente sobre la variable dependiente fue la prueba de diferencia de proporciones y el Chi cuadrado, asumiendo un nivel de confianza del $95 \%$.

La presente investigación no comprometió ni afectó la moral y la ética de las mujeres climatéricas atendidas en el Hospital "Nuestra Señora de las Mercedes" de Carhuaz, ya que antes de su participación se les informó con todo detalle cada uno de los pasos a seguir durante el desarrollo del estudio, así como los beneficios potenciales que se obtendrían al finalizar la investigación; siendo como requisito previo la firma voluntaria de la declaración de consentimiento informado que realizó cada una de las mujeres. Asimismo, es importante aclarar que, al establecer las pautas iniciales de esta investigación desde el punto de vista ético, se consideró seguir como principio general, el respeto a los derechos de las personas con la finalidad de salvaguardar su integridad personal; por lo que se optó por ceñirse estrictamente a las recomendaciones de la Declaración de Helsinki publicada por la Asociación Médica Mundial (LIX Asamblea Médica Mundial en Seoul, Korea) (24).

\section{RESULTADOS}

Al evaluar los síntomas vasomotores dentro del grupo experimental se encontró una diferencia significativa $(p<0,05)$ entre los resultados del pre-test y post-test, evidenciando un incremento en el porcentaje de mujeres asintomáticas del 3,3\% al $33,4 \%$; mientras que todas las mujeres con intensidad severa lograron superar esta situación. Por otro lado, en grupo control la diferencia no fue tan marcada $(p=0,902)$, siendo la variación de la proporción de usuarias asintomáticas de $6,7 \%$ a 10\%. (Tabla 1)

Dentro de los síntomas psicológicos se encontraron cambios significativos $(p<0,05)$ luego de la aplicación del programa de psicoprofilaxis del climaterio, encontrándose que la proporción de mujeres con sintomatología severa disminuyó del 10\% al $0 \%$. Por otro lado, se muestra una escasa diferencia $(p=0,85)$ entre los resultados del pre-test y post-test en el grupo control, donde además la sintomatología moderada con respecto a los problemas psicológicos fue una queja común tanto en el pre-test como en el post-test, con el $13,3 \%$ y $20 \%$, respectivamente. (Tabla 2) 
Tabla 1. Intensidad de los síntomas vasomotores en mujeres climatéricas atendidas en el Hospital "Nuestra Señora de las Mercedes", Carhuaz, Perú.

\begin{tabular}{|c|c|c|c|c|c|c|c|}
\hline & \multicolumn{7}{|c|}{ Evaluación } \\
\hline & \multirow[t]{2}{*}{$\begin{array}{l}\text { Intensidad de } \\
\text { síntomas } \\
\text { vasomotores }\end{array}$} & \multicolumn{2}{|c|}{$\begin{array}{c}\text { Antes } \\
\text { (Pre-test) }\end{array}$} & \multicolumn{2}{|c|}{$\begin{array}{l}\text { Después } \\
\text { (Post-test) }\end{array}$} & \multirow[t]{2}{*}{$\mathrm{Chi}^{2}$} & \multirow[t]{2}{*}{$p^{\dagger}$} \\
\hline & & $\mathrm{n}$ & $\%$ & $\mathrm{n}$ & $\%$ & & \\
\hline \multirow{4}{*}{$\begin{array}{l}\text { Con aplicación de } \\
\text { psicoprofilaxis del } \\
\text { climaterio (grupo } \\
\text { experimental) }\end{array}$} & Asintomática & 1 & 6,6 & 10 & 66,8 & \multirow{4}{*}{17,530} & \multirow{4}{*}{$0,001^{\prime}$} \\
\hline & Leve & 2 & 13,4 & 4 & 26,6 & & \\
\hline & Moderada & 7 & 46,6 & 1 & 6,6 & & \\
\hline & Severa & 5 & 53,4 & 0 & 0,0 & & \\
\hline \multirow{4}{*}{$\begin{array}{c}\text { Sin aplicación de } \\
\text { psicoprofilaxis del } \\
\text { climaterio (grupo } \\
\text { control) }\end{array}$} & Asintomática & 2 & 13,4 & 3 & 20,0 & \multirow{4}{*}{0,577} & \multirow{4}{*}{0,902} \\
\hline & Leve & 3 & 20,0 & 4 & 26,6 & & \\
\hline & Moderada & 6 & 40,0 & 5 & 33,4 & & \\
\hline & Severa & 4 & 26,6 & 3 & 20,0 & & \\
\hline
\end{tabular}

${ }^{\dagger}$ Evaluado mediante la prueba Chi Cuadrado de Stuart-Maxwell

* Variación significativa $(p<0,05)$

Al evaluar la intensidad de los síntomas genitourinarios se observa una clara diferencia $(p<0,001)$ entre los resultados del pre-test y post-test, evidenciándose que todas las mujeres con intensidad severa de problemas genitourinarios (10\%) lograron superar esta situación. Mientras que en el grupo control se evidencia que no existieron diferencias importantes $(p=0,705)$ con respecto a la intensidad de los síntomas genitourinarios, observándose que la mayoría de las mujeres tuvo una intensidad moderada de dicha sintomatología en las dos evaluaciones realizadas. (Tabla 3 )
Se encontró cambios significativos $(p<0,001)$ en la intensidad de los síntomas generales en mujeres climatéricas luego de la aplicación del programa de psicoprofilaxis del climaterio, hallando que la proporción de mujeres con sintomatología moderada y severa disminuyó en ambos casos del 6,7\% y 16,7\% respectivamente, al $0 \%$. Por otro lado, se observó una escasa diferencia $(p=0,543)$ entre los resultados del pre-test y post-test en el grupo sin intervención, donde además la intensidad severa fue una queja constante tanto durante todo el proceso, disminuyendo solo del $20 \%$ al $13,3 \%$. (Tabla 4 )

Tabla 2. Intensidad de los síntomas psicológicos en mujeres climatéricas atendidas en el Hospital "Nuestra Señora de las Mercedes", Carhuaz, Perú.

\begin{tabular}{|c|c|c|c|c|c|c|c|}
\hline & \multicolumn{7}{|c|}{ Evaluación } \\
\hline & \multirow[t]{2}{*}{$\begin{array}{l}\text { Intensidad de } \\
\text { síntomas } \\
\text { psicológicos }\end{array}$} & \multicolumn{2}{|c|}{$\begin{array}{c}\text { Antes } \\
\text { (Pre-test) }\end{array}$} & \multicolumn{2}{|c|}{$\begin{array}{l}\text { Después } \\
\text { (Post-test) }\end{array}$} & \multirow[t]{2}{*}{$\mathrm{Chi}^{2}$} & \multirow[t]{2}{*}{$p^{\dagger}$} \\
\hline & & $\mathrm{n}$ & $\%$ & $\mathrm{n}$ & $\%$ & & \\
\hline \multirow{4}{*}{$\begin{array}{l}\text { Con aplicación de } \\
\text { psicoprofilaxis del } \\
\text { climaterio (grupo } \\
\text { experimental) }\end{array}$} & Asintomática & 1 & 6,6 & 12 & 80,0 & \multirow{4}{*}{16,885} & \multirow{4}{*}{$0,001^{*}$} \\
\hline & Leve & 7 & 46,6 & 2 & 13,4 & & \\
\hline & Moderada & 4 & 26,8 & 1 & 6,6 & & \\
\hline & Severa & 3 & 20,0 & 0 & 0,0 & & \\
\hline \multirow{4}{*}{$\begin{array}{c}\text { Sin aplicación de } \\
\text { psicoprofilaxis del } \\
\text { climaterio (grupo } \\
\text { control) }\end{array}$} & Asintomática & 2 & 13,4 & 1 & 6,6 & \multirow{4}{*}{0,800} & \multirow{4}{*}{0,85} \\
\hline & Leve & 8 & 53,4 & 7 & 46,6 & & \\
\hline & Moderada & 4 & 26,6 & 6 & 40,0 & & \\
\hline & Severa & 1 & 6,6 & 1 & 6,8 & & \\
\hline
\end{tabular}

${ }^{\dagger}$ Evaluado mediante la prueba Chi Cuadrado de Stuart-Maxwell

* Variación significativa 
Tabla 3. Intensidad de los síntomas genitourinarios en mujeres climatéricas atendidas en el Hospital "Nuestra Señora de las Mercedes", Carhuaz, Perú.

\begin{tabular}{|c|c|c|c|c|c|c|c|}
\hline & \multicolumn{7}{|c|}{ Evaluación } \\
\hline & \multirow{2}{*}{$\begin{array}{l}\text { Intensidad de } \\
\text { síntomas } \\
\text { genitourinarios }\end{array}$} & \multicolumn{2}{|c|}{$\begin{array}{c}\text { Antes } \\
\text { (Pre-test) }\end{array}$} & \multicolumn{2}{|c|}{$\begin{array}{l}\text { Después } \\
\text { (Post-test) }\end{array}$} & \multirow[t]{2}{*}{$\mathrm{Chi}^{2}$} & \multirow[t]{2}{*}{$p^{\dagger}$} \\
\hline & & $\mathrm{n}$ & $\%$ & $\mathrm{n}$ & $\%$ & & \\
\hline \multirow{4}{*}{$\begin{array}{l}\text { Con aplicación de } \\
\text { psicoprofilaxis del } \\
\text { climaterio (grupo } \\
\text { experimental) }\end{array}$} & Asintomática & 0 & 0,0 & 11 & 73.4 & \multirow{4}{*}{18,103} & \multirow{4}{*}{$<0,001^{*}$} \\
\hline & Leve & 2 & 13,4 & 1 & 6,6 & & \\
\hline & Moderada & 10 & 66,6 & 3 & 20,0 & & \\
\hline & Severa & 3 & 20,0 & 0 & 0,0 & & \\
\hline \multirow{4}{*}{$\begin{array}{l}\text { Sin aplicación de } \\
\text { psicoprofilaxis del } \\
\text { climaterio (grupo } \\
\text { control) }\end{array}$} & Asintomática & 1 & 6,6 & 0 & 0,0 & \multirow{4}{*}{1,402} & \multirow{4}{*}{0,705} \\
\hline & Leve & 3 & 20,0 & 4 & 26,6 & & \\
\hline & Moderada & 9 & 60,0 & 8 & 53,4 & & \\
\hline & Severa & 2 & 13,4 & 3 & 20,0 & & \\
\hline
\end{tabular}

${ }^{\dagger}$ Evaluado mediante la prueba Chi Cuadrado de Stuart-Maxwell

* Variación significativa

Finalmente se observó una marcada diferencia $(p<0.001)$ entre los resultados del pre-test y post-test de las mujeres climatéricas del grupo experimental con respecto a la presencia de los signos clínicos, evidenciándose una disminución importante en el porcentaje de mujeres con dichos signos (del $46,7 \%$ al $13,3 \%$ ). Sin embargo, en el grupo control no existió diferencias significativas $(p=0,624)$ entre los resultados del pre-test y post-test, observándose que la mayoría de mujeres evidenció dichos signos en ambas evaluaciones (43,3\% y $40 \%$, respectivamente).

(Tabla 5)

\section{DISCUSIÓN}

La evaluación de la influencia de la aplicación de un programa de psicoprofilaxis del climaterio en la intensidad de los síntomas vasomotores de mujeres climatéricas mostraron un incremento en el porcentaje de mujeres asintomáticas; al respecto Fernandes, en Portugal, evidenció que los síntomas vasomotores son uno de los pocos que parecen ser reconocidos por las usuarias como consecuencia de los cambios hormonales (25); hecho que quedó demostrado en el estudio de González, en Cuba, quien encontró que antes de aplicar su intervención

Tabla 4. Intensidad de los síntomas generales en mujeres climatéricas atendidas en el Hospital "Nuestra Señora de las Mercedes", Carhuaz, Perú.

\begin{tabular}{|c|c|c|c|c|c|c|c|}
\hline & \multicolumn{7}{|c|}{ Evaluación } \\
\hline & \multirow[t]{2}{*}{$\begin{array}{l}\text { Intensidad de } \\
\text { síntomas } \\
\text { generales }\end{array}$} & \multicolumn{2}{|c|}{$\begin{array}{c}\text { Antes } \\
\text { (Pre-test) }\end{array}$} & \multicolumn{2}{|c|}{$\begin{array}{l}\text { Después } \\
\text { (Post-test) }\end{array}$} & \multirow[t]{2}{*}{$\mathrm{Chi}^{2}$} & \multirow[t]{2}{*}{$p^{\dagger}$} \\
\hline & & $\mathrm{n}$ & $\%$ & $\mathrm{n}$ & $\%$ & & \\
\hline \multirow{4}{*}{$\begin{array}{l}\text { Con aplicación de } \\
\text { psicoprofilaxis del } \\
\text { climaterio (grupo } \\
\text { experimental) }\end{array}$} & Asintomática & 0 & 0,0 & 12 & 80,0 & \multirow{4}{*}{21,273} & \multirow{4}{*}{$<0,001^{\prime}$} \\
\hline & Leve & 8 & 53,2 & 3 & 20,0 & & \\
\hline & Moderada & 2 & 13,4 & 0 & 0,0 & & \\
\hline & Severa & 5 & 33,4 & 0 & 0,0 & & \\
\hline \multirow{4}{*}{$\begin{array}{l}\text { Sin aplicación de } \\
\text { psicoprofilaxis del } \\
\text { climaterio (grupo } \\
\text { control) }\end{array}$} & Asintomática & 0 & 0,0 & 1 & 6,8 & \multirow{4}{*}{2,144} & \multirow{4}{*}{0,543} \\
\hline & Leve & 7 & 46,6 & 6 & 40,0 & & \\
\hline & Moderada & 2 & 13,4 & 4 & 26,6 & & \\
\hline & Severa & 6 & 40,0 & 4 & 26,6 & & \\
\hline
\end{tabular}

${ }^{\dagger}$ Evaluado mediante la prueba Chi Cuadrado de Stuart-Maxwell

* Variación significativa 
Tabla 5. Presencia de signos clínicos en mujeres climatéricas atendidas en el Hospital "Nuestra Señora de las Mercedes", Carhuaz, Perú.

\begin{tabular}{|c|c|c|c|c|c|c|c|}
\hline & \multicolumn{7}{|c|}{ Evaluación } \\
\hline & \multirow[t]{2}{*}{$\begin{array}{l}\text { Presencia de } \\
\text { signos clínicos }\end{array}$} & \multicolumn{2}{|c|}{$\begin{array}{c}\text { Antes } \\
\text { (Pre-test) }\end{array}$} & \multicolumn{2}{|c|}{$\begin{array}{l}\text { Después } \\
\text { (Post-test) }\end{array}$} & \multirow[t]{2}{*}{$\mathrm{Chi}^{2}$} & \multirow[t]{2}{*}{$p^{\dagger}$} \\
\hline & & $\mathrm{n}$ & $\%$ & $\mathrm{n}$ & $\%$ & & \\
\hline \multirow{2}{*}{$\begin{array}{l}\text { Con aplicación de } \\
\text { psicoprofilaxis del } \\
\text { climaterio (grupo } \\
\text { experimental) }\end{array}$} & Presente & 14 & 93,4 & 4 & 26,6 & \multirow{2}{*}{13,889} & \multirow{2}{*}{$<0,001^{*}$} \\
\hline & Ausente & 1 & 6,6 & 11 & 73,4 & & \\
\hline \multirow{2}{*}{$\begin{array}{c}\text { Sin aplicación de } \\
\text { psicoprofilaxis del } \\
\text { climaterio (grupo } \\
\text { control) }\end{array}$} & Presente & 13 & 86,6 & 12 & 80,0 & \multirow{2}{*}{0,240} & \multirow{2}{*}{0,624} \\
\hline & Ausente & 2 & 13,4 & 3 & 20,0 & & \\
\hline
\end{tabular}

educativa, el $76,6 \%$ reconocieron los sofocos, un $73,3 \%$ las sudoraciones y un $60 \%$ las palpitaciones como signos y síntomas; sin embargo, después de la intervención educativa se logró que el $100 \%$ de las pacientes identificaran las identificaran como cambios vasomotores (26). Coutinho, por su parte, evidenció en Brasil que las mujeres postmenopáusicas que manifestaron mareos y sudoración excesiva presentaban menos actividad física. Así mismo evidenció la reducción de síntomas de la menopausia cuando la actividad diaria se encontraba entre 50 a 100 minutos (27); resultado similar al presentado por Paredes, en Perú, donde el ejercicio aeróbico tuvo un impacto positivo sobre la reducción de los síntomas vasomotores en mujeres postmenopáusicas (28).

Por otro lado, con respecto a los síntomas psicológicos de mujeres climatéricas, se observaron cambios importantes en la intensidad de los síntomas luego de la aplicación del programa de psicoprofilaxis del climaterio, encontrándose que la proporción de mujeres con sintomatología severa disminuyó a un $0 \%$. Lo manifestado guarda relación con lo hallado por Robles en España, quien observó una tendencia al descenso en los valores referidos a tensión, depresión, angustia, fatiga y confusión después de la aplicación de un programa de ejercicio aeróbico, siendo estadísticamente significativo el descenso en la tensión, depresión y angustia (29); mientras que González, en Cuba, encontró que después de la aplicación de una intervención educativa se logró que todas de las mujeres identificaran la irritabilidad, la depresión, nerviosismo y la disminución de la libido como consecuencias propias del periodo del climaterio (26). Asimismo, Lisboa en Brasil reportó la necesidad de estrategias de apoyo psicológico, las cuales deben ser instituidas en el contexto de las políticas de salud (30), conclusión también compartida por Espinoza en Perú, en donde el sentirse conforme físicamente, el temor frente a los cambios en la menopausia, el aprecio y apoyo por parte de la pareja y/o hijos se relacionan significativamente con la intensidad del síndrome climatérico (31).

Al estudiar la influencia de la intervención sobre la intensidad de los síntomas genitourinarios, se observó una influencia significativa, evidenciándose que todas las mujeres con intensidad severa de problemas genitourinarios lograron superar esta situación. Al respecto no se hallaron estudios que evalúen una intervención, sin embargo, Monterrosa en Colombia encontró que las mujeres con origen quechua tienen una sintomatología climatérica más intensa que la descrita en mujeres hispánicas o europeas, con énfasis en la sintomatología urogenital, las cuales aparecen precozmente y comprometen de forma severa a más del $90 \%$ de las mujeres que están en el rango de los 45 - 49 años (32).

Con respecto a la intensidad de los síntomas generales de mujeres climatéricas, se evidenciaron cambios significativos luego de la aplicación del programa, siendo así que al final no se hallaron 
usuarias con sintomatología severa o moderada. Resultados similares fueron reportados por López en Chile, en donde la mayoría de los síntomas de la menopausia se refirieron a los padecimientos musculares y enfermedades de las articulaciones, seguido por el agotamiento mental y físico. Las mujeres con sedentarismo, hábito de fumar y ausencia de actividades recreativas mostraron peores puntuaciones de calidad de vida (33).

Por otro lado, con respecto a la determinación de la influencia de la intervención en la presencia de signos clínicos en mujeres climatéricas, se evidenció una disminución significativa en el porcentaje de mujeres con dichos signos. Acerca de lo manifestado, Cornejo en Perú reporta que existe relación estadísticamente significativa entre los estilos de vida y los signos clínicos, concluyéndose que los estilos de vida no saludables se asociaron a mayor porcentaje de signos y síntomas climatéricos moderados o muy molestos (34).

Se sugiere a las autoridades de los establecimientos de salud, gobiernos locales y Organizaciones No Gubernamentales (ONG), el desarrollo e implementación de planes de intervención que tomen en cuenta los resultados de esta y otras investigaciones con respecto a la aplicación del programa de psicoprofilaxis del climaterio y sus beneficios potenciales en la reducción de la intensidad de la sintomatología de mujeres climatéricas, a fin de mejorar su calidad de vida y prevenir la aparición de enfermedades. Por otro lado, los profesionales en Obstetricia deben poner en práctica nuevas técnicas educativas con respecto a la orientación y consejería de las mujeres climatéricas, reportando lo estudiado para generar evidencia científica y así garantizar una educación integral en el cuidado de su salud, sin la presencia de tabúes, mitos y creencias.

Por lo mencionado se llega a concluir que la aplicación del programa de psicoprofilaxis del climaterio influye significativamente en la reducción de la intensidad de la sintomatología de mujeres climatéricas en el Hospital "Nuestra Señora de las Mercedes" de Carhuaz, evidenciado en la disminución de la intensidad de los síntomas vasomotores, psicológicos, genitourinarios, generales y clínicos. 


\section{REFERENCIAS}

1. Morales S. Preparación integral para el parto y etapas del ciclo de vida. Lima, Universidad San Martín de Porres; 2012.

2. Paredes N. Aspectos demográficos: Hacia una nueva primavera de la mujer después de los 40. Lima: Cimagraf; 2008

3. Blumel J, Roncagliolo M, Gramegna G, Tacla X, Sepulverda H. Prevalencia de síntomas psíquicos y vasomotores en diferentes períodos del climaterio. Rev Chil Obstet Ginecol. 1997; 62: 412-8. [Link]

4. Paredes N. Climaterio y metabolismo del calcio. Rev Med Moderna. 1993; 1: 20 25.

5. Baker A, Simpson S, Dawson D. Sleep disruption and mood changes associated with menopause. J Psychosom Res. 1997; 43: 359-69. [Link]

6. Villaverde C, Araujo E, Cruz F, Roa J, Barbosa W, Ruiz G. Quality of life of rural menopausal women in response to a customized exercise programme. J Advanc Nurs. 2006; 54: 11-9. [Link]

7. Parry B, Martínez L, Maurer E, Lopez A, Sorenson D, Meliska C. Sleep, rhythms and won Part II. Menopause. Sleep Med Rev. 2006; 10: 197-208. [Link]

8. Hay A, Bancroft J, Johnstone E. Affective symptoms in women attending a menopause clinic. Br J Psychiatry. 1994; 164: 513-16. [Link]

9. Chedraui $P$, Blümel JE, Baron $G$, Belzares E, Bencosme A, Calle A, Danckers L, Espinoza MT, Flores D, Gomez G, Hernandez-Bueno JA, Izaguirre $\mathrm{H}$, Leon-Leon $\mathrm{P}$, Lima $\mathrm{S}$, Mezones-Holguin E, Monterrosa A, Mostajo D, Navarro D, Ojeda E, Onatra W, Royer M, Soto E, Tserotas K. Impaired quality of life among middle aged women: a multicentre Latin American study. Maturitas 2008; 61(4): 323-329. [Link]

10. Schnatz PF, Serra J, O'Sullivan DM, Sorosky JI. Menopausal symptoms in Hispanic women and the role of socioeconomic factors. Obstet Gynecol Surv. 2006; 61: 187-93. [Link]
11. Paredes N. Fitoestrógenos: ¿La alternativa de los estrógenos? Rev Per Climat. 2001; 3: 7-10.

12. Dirección del Centro de Salud San Francisco. Implementación de programa de psicoprofilaxis del climaterio. Tacna: CLAS S. C. San Francisco; 2008.

13. Nuñez de Villavicencio F. Psicología y salud. La Habana, Editorial Ciencias Médicas; 2006. [Link]

14. Elavsky S, McAuley E. Physical activity, symptoms, esteem, and life satisfaction during menopause. Maturitas. 2005; 52 374-385. [Link]

15. Nelson DB, Sammel MD, Freeman EW, Lin H, Gracia CR, Schmitz KR. Effect of physical activity on menopausa symptoms among urban women. Med Sci Sports Exerc. 2008; 40: 50-58. [Link]

16. Aiello EJ, Yasui Y, Tworoger SS, Wlrich CM, Irwin ML, Bowen D, et al. Effect of a yearlong, moderate-intensity exercise intervention on the occurrence and severity of menopause symptoms in postmenopausal women. Menopause. 2004; 11: 382-388. [Link]

17. Lindh-Åstrand L, Nedstrand E, Wyon Y, Hammar M. Vasomotor symptoms and quality of life in previously sedentary postmenopausal women randomised to physical activity or estrogen therapy. Maturitas. 2004; 48: 97-105. [Link]

18. Moriyama CK, Oneda B, Bernardo FR Cardoso CGJr, Forjaz CLN, Abrahao SB, et al. A randomized, placebo-controlled trial of the effects of physical exercises and estrogen therapy on health-related quality of life in postmenopausal women. Menopause. 2008; 15: 613-8.

19. Whitcomb BL, Whiteman MK Langenberg P, Flaws JA, Romani WA Physical activity and risk of hot flash among women in midlife. J Women's Health. 2007; 16: 124-133. [Link]

20. Daley AJ, Stokes-Lampard HJ MacArthur C. Exercise to reduce vasomotor and other menopausa symptoms: A review. Maturitas. 2009; 63: 176-180. [Link]
21. Chattha R, Nagarathna R, Padmalatha V, Nagendra HR. Effect of yoga on cognitive functions in climacteric syndrome: a randomised control study. BJOG. 2008; 115: 991-1000. [Link]

22. Servicio de Estadística. Reporte estadístico 2015. Carhuaz: Dirección Ejecutiva del Hospital "Nuestra Señora de las Mercedes"; 2015.

23. Artiles L, Manzano B, Navarro Despaigne D. Cuerpo, sexualidad y climaterio: La necesidad del manejo médico, social e integral. Rev Centroamericana de Obstet y Ginecol. 2008; 1: 27.

24. World Medical Association. Declaración de Helsinki. Finlandia: WMA; 2010.

25. Fernandes A. Menopause and midlife: menopausal symptoms, body weight and well-being [Tesis doctoral]. Lisboa: Instituto Universitário de Ciências Psicológicas, Sociais e da Vida ISPA; 2011.

26. González Y, Hernández I, Hidalgo S, Pedroso J, Feal N, Báez E. Intervención educativa para elevar conocimientos sobre climaterio y menopausia. Revista Archivo Médico de Camagüey. 2012 16(1): 5-14. [Link]

27. Coutinho A, Baptista F. Relationship between physical activity and menopausal symptoms. Revista Brasileira de Atividade Física \& Saúde. 2011; 16(2): 144-49. [Link]

28. Paredes N. Beneficio del ejercicio aeróbico sobre los síntomas vasomotores de pacientes postmenopáusicas. Horiz Med. 2013; 13(3): 15-24. [Link]

29. Robles G. Efectos de un programa de ejercicio aeróbico sobre el estado de ánimo de mujeres menopáusicas. Kronos Actividad Física y Salud. 2008; 7(14): 55-8. [Link]

30. Lisboa L, Saldanha M, Medeiros $P$ Pereira M, Dantas G. Prevalência de transtornos mentais comuns e avaliação da qualidade de vida no climatério. Rev Assoc Med Bras. 2007; 53(5): 414-20. [Link] 
31. Espinoza R. Factores asociados a la intensidad del síndrome climatérico en mujeres que asisten al Hospital Nacional "Hipólito Unanue" durante el primer trimestre del 2015 [Tesis licenciatura]. Lima: Universidad Nacional Mayor de San Marcos; 2015. [Link]

32. Monterrosa A, Romero I, Paternina A, Ojeda E. La elevada prevalencia y severidad de síntomas urogenitales de mujeres indígenas zenúes colombianas en postmenopausia, también es evidenciada en otra población amerindia. Medicina. 2010; 32(4): 295307. [Link]

33. López F, Soares D. Síntomas climatéricos y calidad de vida de usuarias de consultorios de atención primaria de salud, Santiago. Rev Med Chile. 2011; 139 (5): 618-624. [Link]

34. Cornejo V. Relación de los estilos de vida y los síntomas climatéricos en mujeres perimenopáusicas atendidas en el Hospital San Juan de Lurigancho 2014 [Tesis licenciatura]. Lima: Universidad Nacional Mayor de San Marcos; 2015. [Link] 